\title{
A model to follow the evolution in time of free beach fill
}

\author{
L. Rebaudengo Landò \& S. Stura \\ Department of Civil Environmental and Architectural Engineering, \\ Genoa University, Italy
}

\begin{abstract}
In this paper the evolution in time of free beach nourishment is dealt with by considering in a linked manner the processes of longshore diffusion of the filled sand and evolution of the cross profile, which presents an initial beach scarp that of steeper than that of equilibrium. After examining existing models relevant to the single processes, a simplified engineering model is proposed that follows the evolution in successive steps. Each step refers to the actual values of wave condition and profile slope that affect both processes. Application of the model to a realistic beach nourishment supplies an indication of the reduction in time of the surface of the sand platform between the initial boundaries, with particular reference to the first months after the filling works. Final considerations are made about the importance each process holds in the evolution.

Keywords: beach fill, free nourishment, longshore diffusion, cross-shore profile evolution.
\end{abstract}

\section{Introduction}

This paper takes into consideration the changes that a beach nourishment presents over time after a free beach fill is carried out to increase, for a designed extension, the width of the emerged beach, in order to defend existing coastal structures or to increase the space available as a seaside resort. Free fills, built with sand slightly coarser than the native one to improve the stability of the beach without altering its natural features, are appropriate when wave attacks are predominantly head on. Under this hypothesis the background transport rate has no influences, thus is not taken into consideration in the proposed model. In 
addition the tide oscillation is assumed not significant so that the mean sea level can be taken as fixed.

The most usual building procedure utilizes mechanical equipment which arrange for the spreading and regularisation of the sand. An almost horizontal platform is therefore created with a longitudinal extension (length) usually of the order of some kilometres, a width of the order of several ten of metres, height above the mean sea level (berm height) of 1.5-2 metres, with a slope of about $1 / 20$ and water depth at the toe of 1-2 metres. The initial slope of the beach scarp is steeper than that corresponding to the natural equilibrium profile which in turn depends on the characteristics of the borrowed sand.

The new layout, due to the artificial advance toward the sea of the beach front and due to the new profile, brings about a deep modification of the previous littoral dynamics. There results a rapid reduction in the extension of the platform due both to the strong cross-shore transport toward the sea, with a progressive decrease of the slope of the initial profile (process of evolution of the cross profile), and to a gradient of the longshore transport that, starting from the discontinuity at the unprotected boundaries, spreads the sand towards the lateral beaches (process of longshore diffusion).

Before starting any construction of a beach fill, it is convenient to supply the authority concerned with an indication of the reduction of the platform expected in time. Usually these indications are referred to lengthy times, of the order of years, even if the transformations are more evident in the first period immediately after construction. Indeed, while the process of longshore diffusion evolves slowly, the process of evolution of the cross profile appears quickly, under the effect of the first sea storms. This is also the reason why the models for the description of the two processes are used separately and the diffusion is evaluated with reference to year-averaged wave conditions.

The fate of beach fill over time has been dealt with by several authors. It is worth remembering the EU-COAST3D project which analysed different cases of beach fill in the European context. The results, reported in a special edition of Coastal Engineering (Hamm and Stive [1]), highlighted difficulties in the interpretation of the field observations, as also reported by Kamphuis [2] in a revision on modern Coastal Engineering. Other authors have pointed out the lack of appropriate models to describe the overall evolution of the platform, especially in the immediate term (Elko and Wang [3] and Karasu et al. [4]).

In section 2 the models available today for longshore diffusion and for evolution of the cross profile are recalled.

In section 3 the engineering model of simple application proposed here is illustrated which, despite deriving from the models available, is suitable to describe the overall evolution in short times after the end of the construction by taking into account the two processes at the same time with reference to actual sea states. The model can be used either to give a justification of the behaviour found for a beach fill, knowing the characteristics of the recorded sea states, or to formulate a forecast of the changes that a planned beach fill might undergo, starting from a forecast of possible expected sea storms. 
In section 4 the proposed model is applied to a littoral stretch and the prevailing importance of the evolution of the cross profile in the first months after the sand supply is highlighted.

\section{Reference models}

\subsection{Models for longshore diffusion}

By effect of longshore diffusion of sand a rectangular-shaped platform placed in front of an initially straight shoreline assumes an almost trapezoid ("bellshaped") shape which extends beyond the initial boundaries and thins down until the littoral comes back to conditions close to the pre-filled ones.

Models for the evolution of the shoreline are usually those, named 1-line, derived from the formula originally introduced by Pelnard-Considère [5] and later developed by several authors, e.g. Dean [6], Hanson and Kraus [7]. The hypotheses are: permanence of the shape of the cross profile, existence of a depth of closure offshore and dependence of the longshore sand transport on the direction of waves relative to the normal to the shoreline. The basic equation is

$$
\frac{\partial y}{\partial t}+\frac{1}{h_{*}+B} \frac{\partial Q_{\ell}}{\partial x}=0
$$

in which $x$ and $y$ are the longitudinal and transversal coordinates of a point on the shoreline at time $t, h^{*}$ is the depth of closure, $B$ the berm height and $Q_{\ell}$ the volumetric transport rate of sediment. The angle between the outward normal to shoreline and y axis being small, as usually assumed, the equation of diffusion is obtained

$$
\frac{\partial y}{\partial t}=G \frac{\partial^{2} y}{\partial x^{2}}
$$

which allows analytical solutions. The longshore diffusivity $G$ is a quantity whose expression depends on that adopted for the volumetric transport rate, being deduced from the derivative of $Q_{\ell}$ with x. In an ideal case of an initially rectangular-shaped platform of length $\ell$ and width $Y_{0}-$ an appropriate assumption is a length of the order of 50 times the width as considered here the width $\mathrm{Y}$ is modified by the following

$$
Y(x, t)=\frac{Y_{0}}{2}\left\{\operatorname{erf}\left[\frac{\ell}{4 \sqrt{G t}}\left(\frac{2 x}{\ell}+1\right)\right]-\operatorname{erf}\left[\frac{\ell}{4 \sqrt{G t}}\left(\frac{2 x}{\ell}-1\right)\right]\right\}
$$

the axis $x$ being on the straight original shoreline, with zero in the central point. It is worth noting that, to obtain with the aforesaid relation appreciable differences between the values of $Y$ and those of $Y_{0}$, it is necessary to consider large values of $t$. The simplified expression for $Q_{\ell}$ obtained under the hypothesis of shallow water (CERC formula, Shore Protection Manual [8]) is 


$$
Q_{\ell}=\frac{K_{\ell} H_{s b}^{5 / 2} \sqrt{g / \kappa}}{16(s-1)(1-P)} \quad \sin \left(2 \vartheta_{b}\right)
$$

where $s$ is the relative density of the sand, $P$ the porosity, $H_{s b}$ the significant wave height at breaking, $\vartheta_{b}$ the angle of attack relative to the shoreline normal, $\kappa$ the ratio, assumed as constant, between the wave height and the depth $h$, and $K_{\ell}$ a dimensionless coefficient of experimental determination, dependent on the grain size. For $G$ the following expression is obtained

$$
G=\frac{K_{\ell} H_{s b}^{5 / 2} \sqrt{g / \kappa}}{8(s-1)(1-P)\left(h_{*}+B\right)} \cos \left(2 \vartheta_{b}\right)
$$

For small $\vartheta_{b}$ the last term is taken as 1 .

In the aim of taking into account, as well as the wave height, also the peak period $T_{p}$, the mean bottom slope in the breaker zone $S$ and the median grain size $d_{50}$, the following expression can be adopted

$$
Q_{\ell}=K_{\ell}^{\prime} H_{s b}^{2} T_{p}^{1.5} S^{0.75} d_{50}{ }^{-0.25} \sin ^{0.6}\left(2 \vartheta_{b}\right)
$$

where $K_{\ell}^{\prime}$ is a new dimensional coefficient of calibration. The above equation, proposed by Kamphuis [9], if compared with that of the CERC, leads to generally smaller volumetric transport rate, in coherence both with field observations and with the results that are obtained adopting more recent predictive formulas (Bayram et al. [10]; van Rijn [11]). Starting from eqn (6) the following expression for $G$ is deduced

$$
G=\frac{2 K_{\ell}^{\prime} H_{s b}{ }^{2} T_{p}^{1.5} S^{0.75} d_{50}{ }^{-0.25}}{\left(h_{*}+B\right)} \frac{0.6}{\sin ^{0.4}\left(2 \vartheta_{b}\right)} \cos \left(2 \vartheta_{b}\right)
$$

An important indicator of the state of the nourishment is the percentage of platform surface remaining at time $t$. To obtain an analytical expression of such function, named $M(t)$, Dean [12] integrates the equation of diffusion, eqn (2), assuming a constant value for $G$, evaluated with reference to an averaged wave height. In the time interval included between the start of the process and the halving of the platform surface Dean suggests the asymptotic expression

$$
M(t)=1-\frac{2}{\sqrt{\pi}} \frac{\sqrt{G t}}{\ell}
$$

Application of this equation with usual wave climates leads to half-lives of the order of ten years; this result once again highlights that the shoreline change due to longshore diffusion, as already observed, develops over long times.

\subsection{Models for the evolution of the cross profile}

The evolution of the beach profile associated with the cross-shore sand transport caused by single sea storms depends, besides on the offshore wave conditions, 
also on the characteristics of the sediments, native and filled, on the initial profile and on the actual storm sea level. Generally reference is made, with different applications, to Kriebel and Dean's model [13] which extends to the dynamic condition the static equilibrium profile concept. The cross-shore sediment transport rate per unit width of beach $q$ results proportional to the difference between the actual energy dissipation per unit volume $D$ defined by

$$
D=\frac{1}{h} \frac{d\left(E C_{g}\right)}{d y},
$$

where $\left(E C_{g}\right)$ is the energy flux per unit width, and the value $D_{e q}$ occurring when the profile has achieved the equilibrium shape. It turns out

$$
q=K\left(D-D_{e q}\right)
$$

where $K$ is a dimensional empirical coefficient difficult to determine. Through the equation of conservation of mass

$$
\frac{\partial h}{\partial t}+\frac{\partial q}{\partial y}=0
$$

the evolution of $h$ from the trend of $q$ is obtained.

The equilibrium profile generally adopted is the one proposed by Dean [14] starting from Brunn's model [15], which links the local depth $h$ with the distance $y$ from the shoreline through the dimensional parameter $A$, which in turn depends on the median grain size $d_{50}$

$$
h=A y^{2 / 3}
$$

The value of $A$ may be given by the empirical equation (Dean [12])

$$
A=0.067 w^{0.44}
$$

where $A$ is in $\mathrm{m}^{1 / 3}$ and $w$, fall velocity of the sediment in $\mathrm{cm} / \mathrm{s}$, is linked with $d_{50}$ through the relation

$$
w=14 d_{50}^{1.1}
$$

with $d_{50}$ in $\mathrm{mm}$. The Dean equilibrium profile is sometimes modified introducing a zone on the beach face with proper constant slope. The two profiles join at the depth at which their slopes are the same.

When this model is applied to the evolution of the cross profile, it is assumed that the volume of sand moved seaward is distributed until the depth of closure and that the shoreward contributions which may be found at the end of sea storms are negligible, due to the great extension of the depositing zone. Knowledge of the time evolution of the cross profile, which reduces the visible extent of the platform, especially in a short time, is of practical interest, but up to today consolidated indications on the time required to reach the equilibrium conditions are not available. 
Recently Karasu et al. [4] proposed a model for control of beach nourishment that adopts, for the evolution of the cross profile, a new simplified scheme which, to be made operative, requires the specification of empirical coefficients. The scheme, used by the authors to interpret small-scale laboratory experiments with monochromatic waves on sand mounds with a high berm, is based on considerations of dimensional analysis which link the volume eroded with the energy of the incident wave, the geometry of the scarp and the characteristics of the sand. The equations proposed, which correlate the derivative in time of the volume removed $V$ to the difference between the actual slope $S$ of the scarp and the equilibrium slope $S_{o}$, are

$$
\begin{gathered}
\frac{d V}{d t}=K_{c} \frac{H^{2} B}{\left(h_{t}+B\right) T}\left(S-S_{o}\right) p \\
\frac{d y}{d t}=\frac{2 h_{t}}{\left(h_{t}+B\right)^{2}} \frac{d V}{d t} \\
\frac{d z}{d t}=\frac{1}{\ell} \frac{d V}{d t}
\end{gathered}
$$

where $d y / d t$ is the moving back of the shoreline in time, $H$ the incident wave height at the beach fill toe, $B$ the berm height, $h_{t}$ the depth at the toe, $d z / d t$ the raising of the seabed, assumed uniform between the toe and the depth of closure $h^{*}$ and $\ell^{*}$ the distance in between. The dimensionless coefficients $K_{c}$ and $p$ must be specified in the conditions under examination. From the results of laboratory tests Karasu et al. have deduced that the parameter $K_{c}$ depends on the grain size and on the initial slope, but not on the wave period. Of this parameter they do not supply any value, not even for the experiments carried out. As regards $p$, they suggest assuming $p=2$ as a reference value.

\subsection{Applications of the models mentioned}

Operative models aimed at quantifying the effect of the processes considered have been proposed by Larson and Kraus [16] and by Work and Dean [17]. These authors, despite recognising that the two processes are interacting in nature, apply the models in a separate way and introduce coefficients deduced from laboratory tests.

For what concerns diffusion, Larson and Kraus propose the 1-line model described in Section 2.1 and apply it to a triangular-shaped platform. The results are qualitatively similar to those obtained with a rectangular platform. For what concerns the evolution of the cross profile, the same authors suggest adopting different models for the four zones into which they divide the whole beach profile. In particular in the surf zone they adopt the equation of equilibrium relative to the balance of energy dissipated per unit of volume with reference to a Dean profile connected to a linear one on the beach face, assuming for $K$ the value $810^{-6} \mathrm{~m}^{4} \mathrm{~N}^{-1}$. As an example the solution is given for a single sea storm described in analytical form. 
Work and Dean [17] apply similar models to monitoring a beach fill. They introduce a model defined by them as 2-line for the longshore diffusion. For what concerns the evolution of the cross profile they still use the Dean's equation of equilibrium and the same beach profile, under the additional hypothesis of shallow water and assuming $K=0.210^{-6} \mathrm{~m}^{4} \mathrm{~N}^{-1}$. They conclude that the model adopted may also describe short-term behaviour, but state that it would not be correct to consider the two processes separately: " ...The next step will be a fully three-dimensional model that accounts for this interaction ...".

With this in mind, Karasu et al. [4] recently proposed a model that links the two processes with one another. For the evolution of the cross profile they adopt their simplified scheme mentioned in Section 2.2. As regards longshore diffusion, the authors integrate directly, with an explicit first-order scheme, the equation of transport of the solid material coupled with Kamphuis' formulation for the volumetric transport rate $Q_{\ell}$ given by eqn (6), in which the slope changes in time depend on the progressive evolution of the profile. The authors apply the model step by step with monochromatic waves. They observe that the slope, which changes due to the cross transport, causes moving back of the berm crest and longshore diffusion. Furthermore, keeping the toe of the beach fill steady, they calculate the moving back of the shore line. The model results 3-line (berm, shoreline and toe). The results obtained by laboratory small-scale tests confirm the validity of the model. Despite not having been tested in field the model from Karasu et al. represents, from a conceptual point of view, an interesting alternative to the models previously mentioned. To make it operative it is necessary to specify the proper value of the coefficient $K_{c}$.

\section{The proposed operative scheme}

The operative scheme proposed here links the two processes of shoreline diffusion and evolution of the cross profile together assuming temporal steps corresponding to a proper succession of sea states.

\subsection{The model adopted for the process of longshore diffusion}

For the process of diffusion reference is made to the 1-line model adopting for $G$ the expression obtained starting from Kamphuis' formula. Eqn (7) is simplified writing off the term in which $\vartheta_{b}$ appears, as the value of this term is slightly lower than one for the small values of $\theta_{b}$ here assumed

$$
G=\frac{2 K_{\ell}^{\prime} H_{s b}{ }^{2} T_{p}^{1.5} S^{0.75} d_{50}{ }^{-0.25}}{\left(h_{*}+B\right)}
$$

The value $K_{\ell}^{\prime}=0.0023 \mathrm{~m}^{1.25} \mathrm{~s}^{-2.5}$ for the calibration coefficient is assumed according to Kamphuis' field and laboratory tests.

To assess the progressive loss of platform surface the simplified expression of $M(t)$ proposed by Dean, given by eqn (8), is adopted, but considering for each 
step the value of $G$ corresponding to the actual values of the wave characteristics and profile slope. In order to use the formula above, in which $G$ is assumed as constant and the origin of the times corresponds to the initial geometry, a scheme is introduced for which, at each time step, a dummy value $t^{*}$ is evaluated which corresponds to the time interval that would have been necessary to produce the effective diffusion of the platform assuming for $G$ the actual value. The scheme is the following: given at the start of the $i$-th step the values of the remaining platform percentage $M_{i-1}$ and of the slope of the cross profile $S_{i-1}$ obtained in the step $i-1-t h$, the value of $G_{i}$ corresponding to the new breaking wave conditions is calculated; therefore the dummy time $t^{*}$, which would have lead to the value $M_{i-1}$, should $G$ have kept a constant value equal to $G_{i}$ is calculated. The value of $M_{i}$ at the end of the $i$-th step is obtained adopting the value $G_{i}$ associated with the time $t^{*}+\Delta t$, according to the equations

$$
\begin{aligned}
& \sqrt{t_{i}^{*}}=\left(1-M_{i-1}\right) \frac{\sqrt{\pi}}{2} \frac{\ell}{\sqrt{G_{i}}} \\
& M_{i}=1-\frac{2}{\sqrt{\pi}} \frac{\sqrt{\left(t_{i}^{*}+\Delta t\right) G}}{\ell}
\end{aligned}
$$

\subsection{The model adopted for the process of evolution of the cross profile}

To assess the evolution of the cross profile the volume eroded in the step under examination is evaluated utilizing eqns (15) and (16) proposed by Karasu et al., here applied with the actual values of wave height at the toe of the fill and the slope obtained at the end of the previous step. The scheme requires expression of the equations in terms of finite differences and introduction of an appropriate value for the calibration coefficient $K_{c}$. To evaluate a proper value for this coefficient a scaling was carried out starting from the results of experiments by Dette et al. [18] to assess, on a large-scale physical model, the moving back and the modelling of beaches of different initial slopes $S_{v}$ under the action of irregular waves with spectral wave height of the order of one metre. Among the slopes adopted by the authors the slope $S_{v}=0.05$ is chosen, with $d_{50}=0.33 \mathrm{~mm}$ to which corresponds an equilibrium slope $S_{o}=0.025$. The values are consistent with those suggested by Creed et al. [19] for free beach nourishments. Making reference to the equations proposed by Karasu et al. and adopting the value $p=2$, for the case mentioned the value $K_{c}=25$ is obtained. To test the variability of $K_{c}$ the case $S_{v}=0.066$ is also examined for which the value $K_{c}=16.5$ was obtained. It can be seen that a decrease of $K_{c}$ as the initial slope increases is consistent with the fact that an increase in the initial slope makes the evolution faster.

For each $\Delta t$, knowing the wave height at the toe of the fill, evaluated taking into account the set-up, the eroded volume and therefore the moving back of the shoreline, the reduction of the platform surface and the slope of the cross profile are all assessed. The scheme adopted is the following: with reference to the $i$-th 
step the equations are written in finite differences form and the moving back $\Delta y_{i}$ and the slope $S_{i}$ are obtained at the end of the step assuming the depth at the beach fill toe as fixed. The formulas adopted are

$$
\begin{gathered}
\Delta y_{i}=2 \Delta t K_{c} \frac{H^{2} B h_{t}}{\left(h_{*}+B\right)^{3} T}\left(S_{i-1}-S_{o}\right)^{p} \\
\frac{1}{S_{i}}=\frac{1}{S_{v}}+\frac{\sum \Delta y_{i}}{h_{t}}
\end{gathered}
$$

where $S_{i-1}$ is the slope at the end of the previous step. The eroded sand volume, which is distributed up to the depth of closure, gives rise to a rising of the sea bottom that is assumed as negligible in the scheme adopted. The process of evolution of the cross profile is applied only when the wave attack corresponds to "erosion". For this assessment the rule of Larson and Krauss [16] is used as it adapts well to the field data; the erosion occurs when

$$
\frac{H_{o}}{L_{o}}<0.00070\left(\frac{H_{o}}{w T_{p}}\right)^{3}
$$

where $H_{o}$ is the mean wave height offshore, $T_{p}$ the associated peak period, $L_{o}$ the corresponding length and $w$ the already mentioned sediment fall velocity.

\section{An example of application of the procedure}

The procedure proposed is applied to the hypothetical case of a free nourishment on a beach exposed mainly to head on waves. After having examined several recent sand fills, for the initial platform the following characteristics are assumed: length $\ell=3,000 \mathrm{~m}$; width $Y=60 \mathrm{~m}$, surface $180,000 \mathrm{~m}^{2}$; berm height $B=2 \mathrm{~m}$; depth at the toe of the fill $h_{t}=1.50 \mathrm{~m}$, slope $S_{v}=0.05$, resulting fill scarp length $30 \mathrm{~m}$; diameter of the deposited sand $d_{50}=0.33 \mathrm{~mm}$. In addition mean natural slope of the sea bottom $S_{o}=0.025$ is considered.

For application of the model, reference will be made to a stretch of beach of the Tuscan coast exposed head on to SW seas (libeccio). The values $H_{o}, T_{p}$ suitable as offshore data are those from the RON buoy of La Spezia. The ones recorded in the year 1998 are assumed because from January to December the data do not show any significant missing information. The values are known at three-hour intervals, thus the same interval is adopted for the time step in the application of the model. For the transfer to the coast of the deepwater data a scheme that takes into account the processes of shoaling and saturation is adopted (Rebaudengo Landò et al. [20]) up to the depth for which the effects of non linearity are not important (Gentile [21]); from this depth up to the breaking point only shoaling is considered.

To evaluate the longshore diffusion the values $h_{b}$ and $H_{s b}$ at breaking are evaluated utilizing Kamphuis' model [22]. To describe the evolution of the cross 
profile, the erosion conditions being verified by eqn (23), the following procedure is adopted: when $h_{b}<h_{t}$, i.e. the breaking takes place on the fill scarp, the wave height corresponding to the depth $h_{t}$ is assumed; for $h_{b}>h_{t}$, the depthdependent wave height is assumed corresponding to the depth at the toe of the fill increased by a set-up value assumed equal to $20 \%$ of the offshore significant wave height (Guza and Thornton [23]).

At the end of the first year after the filling the surface of the platform results $94,200 \mathrm{~m}^{2}$, equal to about $52 \%$ of the original surface of $180,000 \mathrm{~m}^{2}$, with a moving back of the central zone of about $22.5 \mathrm{~m}$. The reduction is due for about $20 \%\left(17,400 \mathrm{~m}^{2}\right)$ to the diffusion - which induces the spreading of nourishment toward the lateral beaches - and for about $80 \%\left(68,400 \mathrm{~m}^{2}\right)$ by the evolution of the cross profile towards the equilibrium one.

Fig. 1 shows the offshore significant wave height $H_{0}$ and the corresponding remaining surface of the platform $R S$ due to the evolution of the cross profile and that due to the two processes together. The figure highlights the fact that the most important transformations are found in the initial phase, mainly due to the strong effect of the evolution of the cross profile. At the end of the year the slope
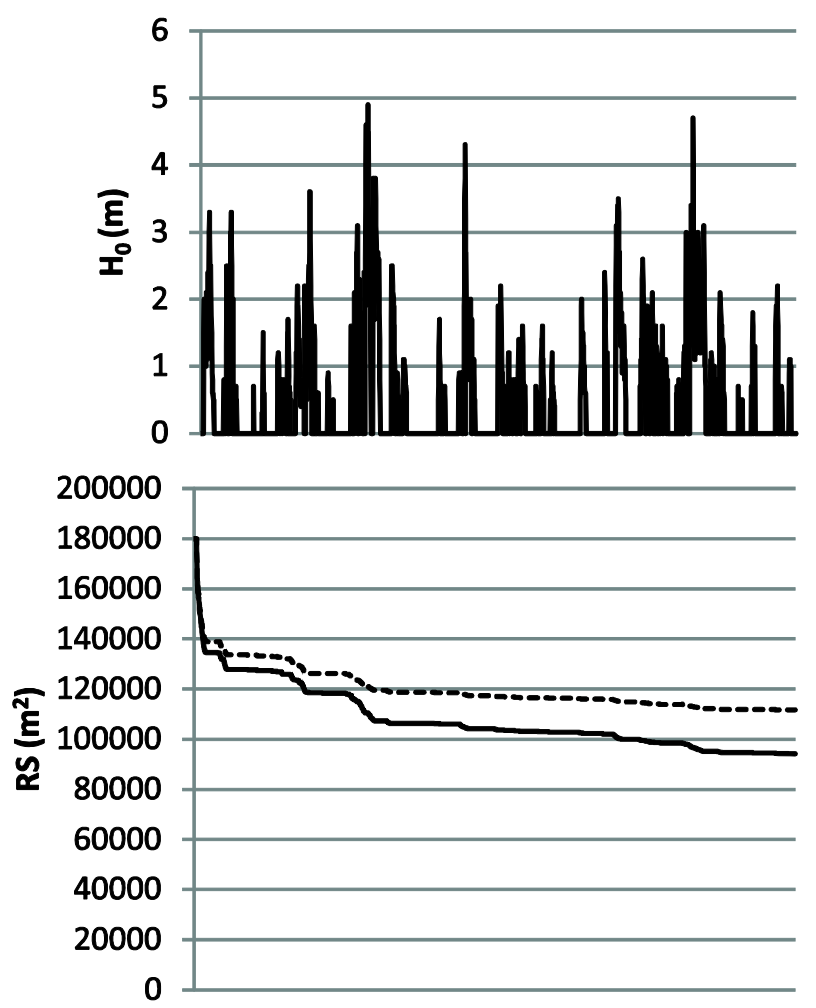

Figure 1: Three-hours values of $H_{0}$ from 01.01 .98 to 31.12 .98 and the relevant values of $R S$ (--- only cross profile evolution). 
of the profile is close to that of equilibrium $(S=0.028$ in comparison with $\left.S_{o}=0.025\right)$ therefore in the following years the evolution of the cross profile will be negligible and the modification due mainly to the process of diffusion.

To outline indicatively the final configuration of the platform, almost "bell shaped", the following first approach scheme is adopted: after having obtained the platform profile corresponding to the surface lost due to diffusion $\left(17,400 \mathrm{~m}^{2}\right)$ by using eqn (8) with a proper constant value of $G$, the final plan profile is obtained by scaling the above profile in order to reach the final surface $\left(94,200 \mathrm{~m}^{2}\right)$. From that procedure a length increased to about 4,500 $\mathrm{m}$ compared with the initial $3,000 \mathrm{~m}$, a final width in the centre of about $37.5 \mathrm{~m}$ compared with the $60 \mathrm{~m}$ of initial fill and a width at the boundaries of about $18 \mathrm{~m}$ are obtained.

To highlight the importance of the initial slope of the front of the fill a small increase of this slope to $S_{v}=0.066$, with $K_{c}=16.5$, is considered, all other conditions being the same. A faster evolution of the cross profile and a quite small variation in diffusion are obtained. The remaining surface at the end of the year considered, instead of $94,200 \mathrm{~m}^{2}$ is reduced to $80,250 \mathrm{~m}^{2}$ corresponding to $45 \%$ of the initial one; this result is due for $18 \%$ by longshore diffusion and for $82 \%$ by the evolution of the cross profile.

\section{Conclusive considerations}

The model proposed allows one to quantify the reduction that a sand platform built for coastal nourishment undergoes in the short term after the end of construction.

The model takes into account the two processes to which the front face of the fill is subjected due to wave attacks: longshore diffusion of the sand beyond the initial boundaries and evolution of the cross slope from the steep initial profile toward the equilibrium one. It has been highlighted that the most marked effects in the initial phase are produced by the evolution of the cross profile.

Fig. 1, relevant to the example presented, shows that the sea storms of the first three months reduces the platform by $34 \%$, of which $87 \%$ due to the evolution of the cross profile and $13 \%$ due to the longshore diffusion; after six months the overall platform reduction is of $45 \%$, of which $83 \%$ due to the evolution of the cross profile and $17 \%$ due to the diffusion; at the end of the year the overall reduction is of $48 \%$, of which $80 \%$ for the evolution of the cross profile and $20 \%$ for the diffusion. These results are in agreement with field observations of the rapid reduction of the platform surface that usually occurs after the sea storms of the first months. With the progressive reduction of the slope of the cross profile the process of diffusion becomes dominant and a slow trend of the shoreline toward the original straight line prevails.

If the calculation were repeated taking into account, as usually done, only longshore diffusion, with the same time history of wave attacks, less realistic results would be obtained because in the first year the moving back of the platform in the central part is not pointed out, thus the scheme, in which in addition the reduction due to diffusion alone is independent of the sequence of 
storm events but rather depends on their cumulative effects as observed by Dean [24], is not appropriate to follow the short time evolution of beach fill, that is the aim of the proposed model.

\section{Acknowledgement}

The study was made possible thanks to a research grant provided by MIUR (n 2008YNPNT9_003)

\section{References}

[1] Hamm L., Stive M. (Ed), Shore nourishment in Europe. Coastal Engineering., Special Issue, 47 (2), pp. 1-264, 2002.

[2] Kamphuis J.W., Coastal engineering - quo vadis?, Coastal Engineering, 53, pp. 133-140, 2006.

[3] Elko N.A. \& Wang P., Immediate profile and platform evolution of a beach nourishment project with hurricane influences, Coastal Engineering, 54, pp. 49-66, 2007.

[4] Karasu S., Work P.A., Cambazoglu M.K. \& Yuksek O., Coupled longshore and cross-shore models for beach evolution at laboratory scale, $J$. of Waterway, Port, Coastal and Ocean Engineering, 121(3), pp. 30-39, Jan./Feb. 30-39 2008.

[5] Pelnard-Considère R., Essai de Theorie de l'Evolution de Formes de Rivage en Plages de Sable et de Galets. IV Journees de l'Hydraulique. Les Energie de la Mèr, Question III Rapport n 1, 1956.

[6] Dean R.G., Principles of beach nourishment, CRC Handbook of Coastal Processes and Erosion. Komar P. D.(Ed), Boca Raton, pp. 217-232, 1983.

[7] Hanson K., Kraus N.C., GENESIS: Generalised Model for Simulating Shoreline Change, Eng. Research Center Vicksburg Tech. Rep., CERC, 1989.

[8] Shore Protection Manual, CERC, Waterways Experiment Station, Vicksburg, USA, 1984.

[9] Kamphuis J.W., Alongshore transport rate of sand,. Proc. of the $28^{\text {th }}$ Int. Conf. on Coastal Engineering, ICCE 2002, pp. 2478-2490, Cardiff, 2002.

[10] Bayram A. Larson M. \& Hanson H., A new formula for the total longshore sediment transport rate, Coastal Engineering, 54, pp. 700-710, 2007.

[11] van Rijn L.C., Longshore sand transport, Proc. of the $28^{\text {th }}$ Int. Conf. on Coastal Engineering, ICCE 2002, pp. 2439-2459, Cardiff, 2002.

[12] Dean R.G., Coastal sediment processes: toward engineering solutions, Proc. Costal Sediments, ASCE, pp. 1-24, 1987.

[13] Kriebel D.L. \& Dean R.G., Numerical simulation of time-dependent beach and dune erosion, Coastal Engineering, 119, pp. 221-245, 1985.

[14] Dean R.G., Equilibrium beach profiles: characteristics and applications, J. of Coastal Research., 7(1), pp. 53-84, 1991.

[15] Bruun P., Coast Erosion and Development of Beach Profiles, U.S.Army Tech. Mem., 44, Vicksburg, 1954. 
[16] Larson M. \& Kraus N., Mathematical modelling of the fate of beach fill, Coastal Engineering, 16, pp.83-114, 1991.

[17] Work P.A. \& Dean R.G., Assessment and prediction of beach nourishment evolution. J. of Waterway, Port, Coastal and Ocean Engineering,121(3), pp. 182-189, May/June 1995.

[18] Dette H.H., Larson M., Murphy J., Newe J., Peters K. Reniers A. \& Steetzel H., Applications of prototype flume tests for beach nourishment assessment, Coastal Engineering, 47, pp. 137-177, 2002.

[19] Creed C.G., Bodge K.R. \& Suter C.L., Construction slopes for beach nourishment projects, J. of Waterway, Port, Coastal and Ocean Engineering, 126(1), pp. 57-62, Jan./Febr, 2000.

[20] Rebaudengo Landò L., Gentile R. \& Scarsi G., A nonlinear spectral model for directional random waves at decreasing depths, J. of Offshore and Polar Engineering, 9(2), pp 81-89, 1999.

[21] Gentile R., A procedure to evaluate the longshore sediment transport due to random waves, Proc. of the $10^{\text {th }}$ Int. Offshore and Polar Engineering Conference, III, pp. 673-680, Seattle, 2000.

[22] Kamphuis, J.K., Incipient wave breaking, Coastal Engineering, 15, pp 185203, 1991.

[23] Guza R.T. \& Thornton E.B., Wave set-up on a natural beach, J. of Geoph. Research, 86 C5, pp. 4133-4137, 1981.

[24] Dean R. G., Beach nourishment projects as large scale experiments in nature. Proc. of the $28^{\text {th }}$ Int. Conf. on Coastal Engineering, ICCE 2002, pp. 3649-3661, Cardiff, 2002. 